\title{
When to Synchronize in Uplink CDMA
}

\author{
Nicolas Bonneau, Eitan Altman \\ MAESTRO, INRIA Sophia Antipolis \\ 2004 Route des Lucioles, B.P. 93 \\ 06902 Sophia Antipolis, France \\ Email: \{nicolas.bonneau, eitan.altman\}@sophia.inria.fr
}

\author{
Mérouane Debbah, Giuseppe Caire \\ Mobile Communications Group, Institut Eurecom \\ 2229 Route des Cretes, B.P. 193 \\ 06904 Sophia Antipolis, France \\ Email: \{merouane.debbah, giuseppe.caire\}@eurecom.fr
}

\begin{abstract}
In this contribution, the performance of an uplink CDMA system with orthogonal spreading is analyzed. A useful framework is provided in order to determine if synchronization of the users gives a significant performance improvement. Using asymptotic arguments, analytical expressions of the spectral efficiency for the Matched Filter and Successive Interference Cancellation Matched Filter are derived. The results, applied in the general case of a multipath channel, provide a simple expression of the cell capacity based only on a few meaningful parameters.
\end{abstract}

\section{INTRODUCTION}

Usual studies of uplink CDMA schemes suppose a multiple access communication scheme where each user modulates his signal with a pseudo-random i.i.d. [1], [2] sequence. One of the reasons relies on the fact that, due to the multipath channel, the convolution of the codes with the different channels of the users can be represented as a new set of codes with properties similar to a pseudo-random sequence due to the randomness of the channel. As a consequence, even if codes are designed for an orthogonal multiple access scheme, the multi-path channel unfortunately destroys orthogonality. However, recently, Debbah et al. [3], [4] showed that, as far as downlink is concerned, a non-negligible gain can be achieved if one uses orthogonal codes to serve the users, especially for highly loaded systems. The intuitive idea is that by appropriate equalization, a user can restore orthogonal access in the network by compensating the effect of his own channel (which is also common to all the users in the downlink). The gain is mainly a function of the load (see [5]) and the type of equalizer. However, in the uplink, such a result can not be applied as each user code is distorted independently by a different channel. As a consequence, the ability of any equalization scheme to restore orthogonality is very limited and is mostly dependent on the channel -orthogonality destroying- fading characteristics. The goal of this contribution is to assess more precisely how multipath affects the performance of uplink-CDMA. In particular, for a given statistical environment, the gain of orthogonalsynchronizing the users will be theoretically quantified with respect to the packet-overhead of signaling. The setting is analyzed for the simple receiver structure of the Matched Filter as well as the Successive Interference Cancellation Matched Filter. In order to obtain interpretable expressions, the problem is analyzed in the asymptotic regime: a high number of users is considered where the spreading length $N$ tends to infinity, the number of users $K$ tends to infinity but the ratio $\frac{K}{N} \rightarrow \alpha$ is constant. In this contribution, the effect of path loss and shadowing, albeit important, is neglected (it is however a straightforward extension of this paper). The results are based on random unitary matrix theory [6], [7]. This tool enables us to express the SINR in a very simple form in the large system limit. Moreover, the theoretical results are shown to be very accurate predictions of the system's behavior in the finite size case (spreading length $N$ of 256 , see section $\mathrm{V}$ ).

This paper is structured as follows. In section II, the CDMA model is introduced together with some results on Random Unitary Matrix Theory. In section III, the SINR (Signal to Interference plus Noise ratio) expression with Matched Filter is derived and discussions are provided in sections IV and V together with simulations. Finally, in section VI, the extension to other receivers is described.

\section{MODEL}

We consider a single CDMA cell (inter-cell interference free case). The spreading length $N$ is fixed. The number of users in the cell is $K$, with the assumption that $K<N$, so that the spreading codes can be chosen mutually orthogonal. The general case of wide-band CDMA is considered where the signal transmitted by user $k$ has complex envelope

$$
x_{k}(t)=\sum_{n} s_{k n} v_{k}(t-n T)
$$

$v_{k}(t)$ is an weighted sum of elementary modulation pulses which satisfy the Nyquist criterion with respect to the chip interval $T_{c}\left(T=N T_{c}\right)$

$$
v_{k}(t)=\sum_{\ell=1}^{N} v_{\ell k} \psi\left(t-(\ell-1) T_{c}\right)
$$

The signal is transmitted over a frequency selective channel with impulse response $c_{k}(\tau)$. Under the assumption of slowlyvarying fading, the continuous time received signal $y(t)$ at the base station has the form:

$$
y(t)=\sum_{n} \sum_{k=1}^{K} s_{k n} \int c_{k}(\tau) v_{k}(t-n T-\tau) d \tau+n(t)
$$

where $n(t)$ is the complex white Gaussian noise. The signal (after pulse matched filtering by $\psi^{*}(-t)$ ) is sampled at the chip rate to get a discrete-time signal that has the form:

$$
\mathbf{y}=\mathbf{C}_{1} \mathbf{v}_{1} s_{1}+\mathbf{C}_{2} \mathbf{v}_{2} s_{2}+\cdots+\mathbf{C}_{K} \mathbf{v}_{K} s_{K}+\mathbf{n}
$$


where $\mathbf{C}_{k}$ are $N \times N$ Toeplitz matrices representing the frequency selective fading for the $k$-th user.

Users are assumed to employ orthogonal codes. Although not restrictive and in order to derive tractable expressions of the SINR, vectors $\mathbf{v}_{k}=\left[v_{1 k}, \ldots, v_{N k}\right]^{T}$ are supposed to be columns extracted from a random unitary matrix $\mathbf{V}$. A random unitary matrix $\mathbf{V}=\left[v_{i k}\right]$ is a matrix with complex entries such as $\mathbf{V} \mathbf{V}^{H}=\mathbf{V}^{H} \mathbf{V}=\mathbf{I}$. Note that the entries are therefore dependent (in comparison with the i.i.d. case of [8]):

$$
\begin{aligned}
& \sum_{k=1}^{N}\left|v_{i k}\right|^{2}=\sum_{i=1}^{N}\left|v_{i k}\right|^{2}=1, \quad \text { for all } 1 \leq i, k \leq N \\
& \sum_{l=1}^{N} v_{i l} v_{l k}^{*}=0, \quad \text { for all } i \neq k
\end{aligned}
$$

The following definition is given in [7]. Since the set $\mathcal{U}(N)$ of $N \times N$ random unitary matrices forms a compact topological group with respect to the matrix multiplication and the usual topology, there exists a unique nonzero left and right invariant measure. It is known as the Haar measure. A random matrix $\mathbf{V}$ is Haar unitary if it takes its values uniformly in $\mathcal{U}(N)$, i.e., if for any subset $H$ of $\mathcal{U}(N)$, the probability that $\mathbf{V} \in H$ is equal to the normalized Haar measure $\mu$ of $H$ :

$$
\mathbb{P}(\mathbf{V} \in H)=\mu(H)
$$

Given that the left invariance characterizes the Haar measure, to show that a unitary random matrix $\mathbf{V}$ is Haar distributed, it is sufficient to show that for any $\mathbf{U} \in \mathcal{U}(N)$, $\mathbf{U V}$ has the same distribution as $\mathbf{V}$. The Gram-Schmidt orthonormalization procedure can be used on the column vectors of a $N \times N$ Gaussian matrix with independent entries to obtain a Haar unitary matrix. If $\mathbf{X}$ is a Gaussian i.i.d. matrix, then $\mathbf{V}=$ $\mathbf{X}\left(\mathbf{X}^{H} \mathbf{X}\right)^{-1 / 2}$ is Haar unitary:

- $\mathbf{V} \mathbf{V}^{H}=\mathbf{X}\left(\mathbf{X}^{H} \mathbf{X}\right)^{-1 / 2}\left(\mathbf{X}^{H} \mathbf{X}\right)^{-1 / 2} \mathbf{X}^{H}=\mathbf{I}$,

- $\mathbf{U V}=\mathbf{U X}\left(\mathbf{X}^{H} \mathbf{U}^{H} \mathbf{U X}\right)^{-1 / 2}$ has the same distribution as $\mathbf{V}$.

Since the users are supposed to be synchronized and for sake of simplicity, we will consider in all the following that users add a cyclic prefix of length equal to the channel impulse response length to their code sequence. This case is similar to uplink MC-CDMA [9], [10] and as a consequence, $\left\{\mathbf{C}_{k}\right\}$ becomes circulant (see [11]) and can be diagonalized in a Fourier basis F. Model (2) simplifies therefore to:

$\mathbf{y}=\mathbf{F} \mathbf{H}_{1} \mathbf{F}^{H} \mathbf{v}_{1} s_{1}+\mathbf{F} \mathbf{H}_{2} \mathbf{F}^{H} \mathbf{v}_{2} s_{2}+\cdots+\mathbf{F} \mathbf{H}_{K} \mathbf{F}^{H} \mathbf{v}_{K} s_{K}+\mathbf{n}$ where $\mathbf{H}_{k}$ is a diagonal matrix with diagonal elements $\left\{h_{i k}\right\}_{i=1 \ldots N}$. For each user $k$, the coefficients $h_{i k}$ are the discrete Fourier transform of the channel impulse response. Since users employ Haar unitary codes and every unitary tranformation of a Haar unitary vector is a Haar unitary vector (so that $\mathbf{w}_{i}=\mathbf{F}^{H} \mathbf{v}_{i}$ has the same distribution as $\mathbf{v}_{i}$ for any $i$ ), one can multiply $\mathbf{y}$ with $\mathbf{F}^{H}$ without any change of the statistics:

$$
\begin{aligned}
\mathbf{y} & =\mathbf{H}_{1} \mathbf{w}_{1} s_{1}+\mathbf{H}_{2} \mathbf{w}_{2} s_{2}+\cdots+\mathbf{H}_{K} \mathbf{w}_{K} s_{K}+\mathbf{n} \\
& =(\mathbf{H} \odot \mathbf{W}) \mathbf{s}+\mathbf{n}
\end{aligned}
$$

where $\odot$ is the Hadamard (element-wise) product. $\mathbf{H}$ is the frequency selective fading matrix, of size $N \times K$ :

$$
\mathbf{H}=\left[\begin{array}{cccc}
h_{11} & h_{12} & \ldots & h_{1 K} \\
\vdots & \vdots & & \vdots \\
h_{N 1} & h_{N 2} & \ldots & h_{N K}
\end{array}\right]
$$

We will suppose in the following that

$$
\forall i, k, \mathbb{E}_{h_{k}}\left[\left|h_{i k}\right|^{2}\right]=\varrho
$$

W is an $N \times K$ orthogonal spreading matrix:

$$
\mathbf{W}=\left[\mathbf{w}_{1}\left|\mathbf{w}_{2}\right| \cdots \mid \mathbf{w}_{K}\right] \text { where } \mathbf{w}_{k}=\left[\begin{array}{c}
w_{1 k} \\
\vdots \\
w_{N k}
\end{array}\right]
$$

Note that asymptotically (as $N \rightarrow \infty$ ), for a given multipath channel of length $L$, model (3) is also valid for the case of uplink DS-CDMA since all Toeplitz matrices can be asymptotically diagonalized in a Fourier Basis (see [12], [13]).

\section{MATCHED FILTER}

Without loss of generality, let us focus on the first user and denote:

$$
\mathbf{H}=\left[\mathbf{h}_{1} \mid \mathbf{H}^{\mathrm{C}}\right], \mathbf{W}=\left[\mathbf{w}_{1} \mid \mathbf{W}^{\mathrm{C}}\right], \mathbf{s}=\left[\begin{array}{l}
s_{1} \\
\mathbf{s}^{\mathrm{C}}
\end{array}\right]
$$

where $\mathbf{h}_{1}$ is the first column of $\mathbf{H}, \mathbf{w}_{1}$ is the first column of $\mathbf{W}$ corresponding to the code of the first user, and $s_{1}$ is the signal of the first user. Supposing perfect channel knowledge at the receiver, the matched filter is given by $\mathbf{g}^{H}=\left(\mathbf{h}_{1} \odot \mathbf{w}_{1}\right)^{H}$. The signal at the matched filter output is given by:

$$
\mathbf{g}^{H} \mathbf{y}=\left|\mathbf{h}_{1} \odot \mathbf{w}_{1}\right|^{2} s_{1}+\mathbf{g}^{H}\left(\mathbf{H}^{\complement} \odot \mathbf{W}^{\complement}\right) \mathbf{s}^{\complement}+\mathbf{g}^{H} \mathbf{n}
$$

This leads to the following expression for $\operatorname{SINR}\left(x_{1}\right)$

$$
\frac{\left(\sum_{i=1}^{N}\left|h_{i 1}\right|^{2}\left|w_{i 1}\right|^{2}\right)^{2}}{\sum_{i=1}^{N}\left|h_{i 1}\right|^{2}\left|w_{i 1}\right|^{2} \sigma^{2}+\sum_{k=2}^{K}\left|\sum_{i=1}^{N} h_{i 1}^{*} h_{i k} w_{i 1}^{*} w_{i k}\right|^{2}}
$$

Proposition 1: For given channel coefficients, the SINR, with orthogonal codes, converges almost surely to a deterministic value as $N \rightarrow \infty$ and $\frac{K}{N} \rightarrow \alpha$, namely:

$$
\operatorname{SINR}^{\text {orth }}\left(x_{1}\right)=\frac{\xi_{1}}{\sigma^{2}+\alpha\left(\varrho-\eta_{1}\right)}
$$

where

$$
\eta_{1}=\frac{\mathbb{E}_{h_{k}}\left[\left|\frac{1}{W} \int_{-\frac{W}{2}}^{\frac{W}{2}} h_{1}(f) h_{k}^{*}(f) d f\right|^{2}\right]}{\frac{1}{W} \int_{-\frac{W}{2}}^{\frac{W}{2}}\left|h_{1}(f)\right|^{2} d f}
$$

and

$$
\xi_{1}=\frac{1}{W} \int_{-\frac{W}{2}}^{\frac{W}{2}}\left|h_{1}(f)\right|^{2} d f
$$

Proof: The proof is in the appendix. 
Note that in the case of Gaussian random i.i.d. codes (i.i.d. elements with zero mean and variance $\frac{1}{N}$ ), the SINR has the following expression (see [8], [14]):

$$
\operatorname{SINR}^{\text {rand }}\left(x_{1}\right)=\frac{\xi_{1}}{\sigma^{2}+\alpha \varrho}
$$

$\eta_{1}$ characterizes the orthogonality gain of the channel and ranges from 0 to $\varrho$. The orthogonality gain is function of the selectivity of the channel as well as the correlation between the channels. For example, if the channels are all the same, SINR $^{\text {orth }}=\frac{\varrho}{\sigma^{2}}$ and the orthogonality gain is maximal.

\section{DISCUSSION}

We consider the particular case of a multipath channel. The model of the channel is given by

$$
c_{k}(\tau)=\sum_{p=0}^{L-1} c_{p k} \delta\left(\tau-\tau_{p k}\right)
$$

where we assume that the channel is invariant during the time considered. In order to compare channels at the same signal to noise ratio, we constrain the fading coefficients to be complex Gaussian i.i.d. random variables with zero mean and variance $\frac{\varrho}{L}$. Usually, coefficients $c_{p k}$ are supposed to be independent with decreasing variance as the delay increases. In all cases, $\sum_{p=0}^{L-1} \mathbb{E}\left[\left|c_{p k}\right|^{2}\right]=\varrho$. For each user $k$, the coefficients $h_{i k}$ are the Discrete Fourier Transform of the fading process. The Fourier transform of $c(\tau)$ is

$$
h_{k}(f)=\sum_{p=0}^{L-1} c_{p k} e^{-j 2 \pi f \tau_{p k}}|\Phi(f)|^{2}
$$

where we assume that the filter $\Phi(f)$ is such that, given the bandwidth $W$,

$$
\Phi(f)= \begin{cases}1 & \text { if }-\frac{W}{2} \leq f \leq \frac{W}{2} \\ 0 & \text { otherwise }\end{cases}
$$

Sampling at the various frequencies $f_{1}=-\frac{W}{2}, f_{2}=-\frac{W}{2}+$ $\frac{1}{N} W, \ldots, f_{N}=-\frac{W}{2}+\frac{N-1}{N} W$, we obtain the coefficients $h_{i k}, 1 \leq i \leq N$, as

$$
h_{i k}=h_{k}\left(f_{i}\right)=\sum_{p=0}^{L-1} c_{p k} e^{-j 2 \pi \frac{i}{N} W \tau_{p k}} e^{j \pi W \tau_{p k}}
$$

It is immediate to check that $h_{i k}$ satisfies (4).

For ease of understanding of the impact of the number of paths on the orthogonality gain, the delays are supposed to be uniformly distributed according to the bandwidth

$$
\tau_{p k}=\frac{p}{W}
$$

As a consequence, we have:

$$
\xi_{1}=\sum_{p=0}^{L-1}\left|c_{p 1}\right|^{2}
$$

and

$$
\begin{array}{r}
\mathbb{E}_{h_{k}}\left[\left|\frac{1}{W} \int_{-\frac{W}{2}}^{\frac{W}{2}} h_{1}(f) h_{k}^{*}(f) d f\right|^{2}\right] \\
=\mathbb{E}_{c_{k}, \tau_{k}}\left[\left|\sum_{p=0}^{L-1} \sum_{q=0}^{L-1} c_{p 1} c_{q k}^{*} \frac{1}{W} \int_{-\frac{W}{2}}^{\frac{W}{2}} e^{-j 2 \pi f\left(\tau_{p 1}-\tau_{q k}\right)} d f\right|^{2}\right] \\
=\mathbb{E}_{c_{k}, \tau_{k}}\left[\left|\sum_{p=0}^{L-1} \sum_{q=0}^{L-1} c_{p 1} c_{q k}^{*} \frac{\sin \pi W\left(\tau_{p 1}-\tau_{q k}\right)}{\pi W\left(\tau_{p 1}-\tau_{q k}\right)}\right|^{2}\right]
\end{array}
$$

Using simplifying hypothesis (6), this is equal to

$$
\begin{aligned}
\mathbb{E}_{c_{k}}\left[\left|\sum_{p=0}^{L-1} c_{p 1} c_{p k}^{*}\right|^{2}\right] & =\mathbb{E}_{c_{k}}\left[\sum_{p=0}^{L-1} \sum_{q=0}^{L-1} c_{p 1} c_{q 1}^{*} c_{p k}^{*} c_{q k}\right] \\
& =\frac{\varrho}{L} \sum_{p=0}^{L-1}\left|c_{p 1}\right|^{2}
\end{aligned}
$$

This gives us the following expressions for the SINR:

$$
\begin{aligned}
\operatorname{SINR}^{\text {orth }}\left(x_{1}\right) & =\frac{\sum_{p=0}^{L-1}\left|c_{p 1}\right|^{2}}{\sigma^{2}+\alpha \varrho\left(1-\frac{1}{L}\right)} \\
\operatorname{SINR}^{\text {rand }}\left(x_{1}\right) & =\frac{\sum_{p=0}^{L-1}\left|c_{p 1}\right|^{2}}{\sigma^{2}+\alpha \varrho}
\end{aligned}
$$

We observe that the orthogonal gain depends only on the four parameters $\sigma^{2}, \alpha, \varrho$ and $L$ :

$$
\frac{\operatorname{SINR}^{\text {orth }}\left(x_{1}\right)}{\operatorname{SINR}^{\text {rand }}\left(x_{1}\right)}=\frac{\sigma^{2}+\alpha \varrho}{\sigma^{2}+\alpha \varrho\left(1-\frac{1}{L}\right)}
$$

Remarkably, at high SNR $\left(\sigma^{2} \rightarrow 0\right)$, the SINR gain is given by:

$$
\frac{\operatorname{SINR}^{\text {orth }}\left(x_{1}\right)}{\operatorname{SINR}^{\text {rand }}\left(x_{1}\right)}=\frac{L}{L-1}
$$

Hence, in the case of a two-path channel, one can increase by $3 \mathrm{~dB}$ the SINR by synchronizing the users whereas for a 5-path channel, the synchronization gain is less than $1 \mathrm{~dB}$.

\section{Simulations}

Simulations (not plotted here due to limited space) show that the theoretical formula for the expression of the SINR gain is very close to curves obtained by generating at random a single fading matrix and a single spreading code matrix for a realistic spreading length of $N=256$.

In figure 1, the mean spectral efficiency of the system with Matched filter has been plotted. For the case of orthogonal spreading, the mean spectral efficiency is given by:

$$
\begin{aligned}
\gamma & =\alpha \mathbb{E}_{c_{p 1}}\left[\log _{2}\left(1+\frac{\sum_{p=0}^{L-1}\left|c_{p 1}\right|^{2}}{\alpha\left(\frac{1}{\gamma \frac{E_{\mathrm{b}}}{N_{0}}}+\varrho\left(1-\frac{1}{L}\right)\right)}\right)\right] \\
& =\alpha \int_{0}^{+\infty} \log _{2}\left(1+\frac{x}{\alpha\left(\frac{1}{\gamma \frac{E_{\mathrm{b}}}{N_{0}}}+\varrho\left(1-\frac{1}{L}\right)\right)}\right) p(x) d x
\end{aligned}
$$




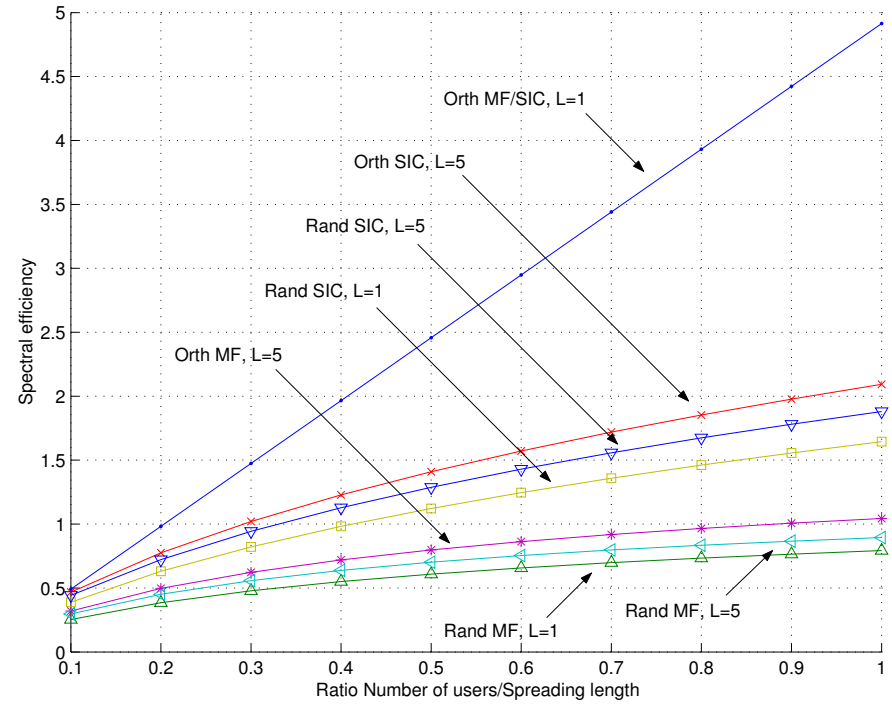

Fig. 1. Spectral efficiency of the multipath channel, SNR $=10 \mathrm{~dB}$

where $x$ represents the random variable $\sum_{p=0}^{L-1}\left|c_{p 1}\right|^{2}$, and $p(x)$ is its distribution, given by a Chi-Squared distribution with $2 L$ degrees of freedom

$$
p(x)=\frac{x^{L-1} e^{-L x / \varrho}}{(L-1) !(\varrho / L)^{L}}
$$

In order to assess the gap with more complex receivers, the performance of the Successive Interference Cancellation (SIC) Matched Filter [15] has been plotted in figure 1 in addition to the simple Matched Filter (MF). The principle of SIC receivers is quite simple: assuming ergodic channels, users are ordered and are decoded successively. At each step, supposing that the user has been encoded at the appropriate decoding rate, the signal is decoded and its contribution to the interference is then perfectly substracted. This removes some of the inter-user interference and therefore increases the SINR of the following decoded users. The SINR of the $k$-th decoded user is then:

$$
\operatorname{SINR}^{\text {orth }}\left(x_{k}\right)=\frac{\sum_{p=0}^{L-1}\left|c_{p k}\right|^{2}}{\sigma^{2}+\frac{K-k+1}{N} \varrho\left(1-\frac{1}{L}\right)}
$$

since the contributions of the $k-1$ first decoded users have already been substracted. In the limit when $N \rightarrow \infty$ and $\frac{K}{N} \rightarrow$ $\alpha, \gamma$ is then given by the implicit equation:

$\gamma=\int_{0}^{\alpha} \int_{0}^{+\infty} \log _{2}\left(1+\frac{x}{y\left(\frac{1}{\gamma \frac{E_{\mathrm{b}}}{N_{0}}}+\varrho\left(1-\frac{1}{L}\right)\right)}\right) p(x) d x d y$

Figure 1 shows $\gamma$ for various values of $L$ with orthogonal codes, with or without successive interference cancellation (SIC), as well as comparative plots of $\gamma$ obtained with random i.i.d. spreading codes. The following results are obtained:

- i.i.d. spreading always provides a lower spectral efficiency than orthogonal spreading, with respect to the same filter.

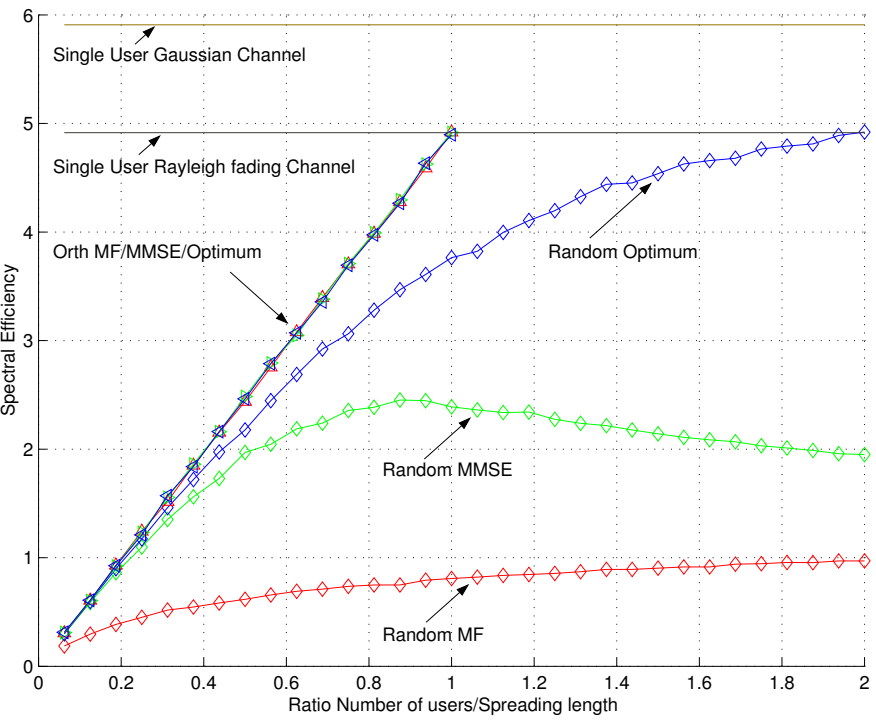

Fig. 2. Simulations on the multipath channel, $\mathrm{L}=1, \mathrm{SNR}=10 \mathrm{~dB}$

- In the case of orthogonal spreading, the spectral efficiency is higher for low values of $L$ for any receiver (to ensure orthogonality between users). This is in contrast with the case of i.i.d. spreading where $L$ must be high to decrease the randomness of the fading.

- As $L$ increases, the gap between orthogonal and i.i.d. spreading reduces for any kind of receiver. This result has already been shown previously through the orthogonality gain expression.

- For $L>2$, the gain of using a SIC scheme with respect to the Matched filter is equivalent for i.i.d. and orthogonal spreading.

\section{EXTENSION TO OTHER RECEIVERS}

For the MMSE and optimum filters, the study involves more sophisticated tools for the orthogonal case and results can be found in [16]. In the case of i.i.d. codes, the results rely on a theorem due to Girko (see [17], [18]).

In order to evaluate the potential gains, simulations are shown for both orthogonal and i.i.d. random codes, with 3 different filters: Matched Filter (MF), MMSE Filter and Optimum Filter, on channels with respectively $\mathrm{L}=1$ path (figure 2) and $L=5$ paths (figure 3). The curves prompt the same comments as figure 1: though there is always a gain in spectral efficiency with orthogonal codes, this gain decreases as $L$ increases for any receiver. However, note that in the particular case of the optimum receiver, i.i.d. codes achieve the single user Gaussian bound when the load tends to infinity. Therefore, i.i.d. codes can outperform the performance of orthogonal codes (if the system is working at high loads, see figure 3 ).

\section{CONCLUSION}

Using asymptotic arguments, an explicit expression of the SINR of an uplink CDMA cell using orthogonal spreading codes and Matched Filter has been derived considering a 


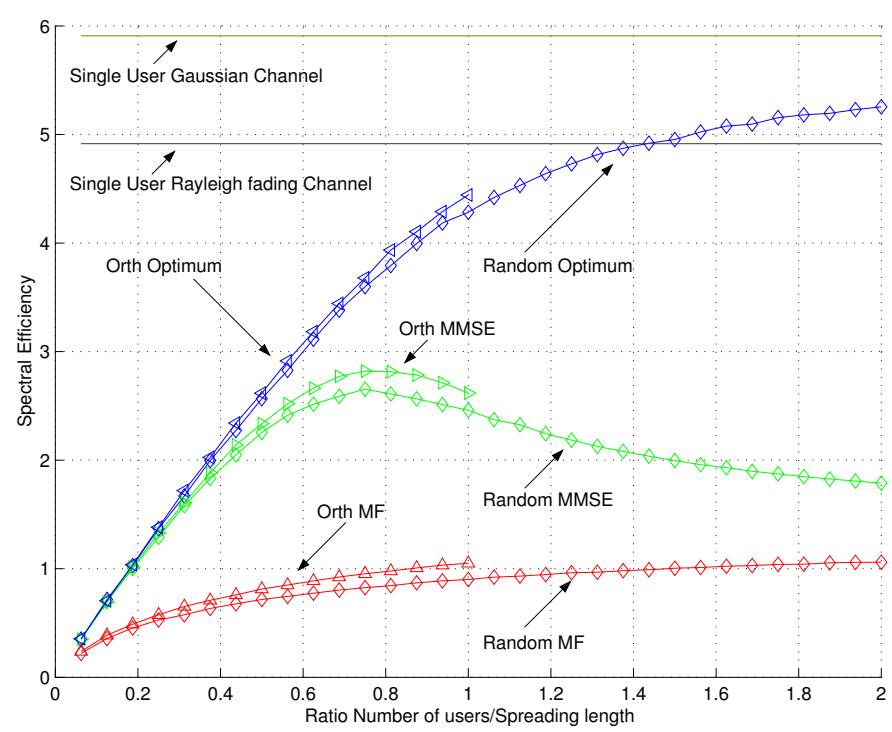

Fig. 3. Simulations on the multipath channel, $\mathrm{L}=5, \mathrm{SNR}=10 \mathrm{~dB}$

realistic frequency selective fading model. The orthogonality gain has been shown to depend mainly on the number of paths and the load of the system through very simple insightful expressions. As a consequence, the need to synchronize the users is mainly a function of the environment at hand and one could think of adaptive synchronization protocols for future multiple access CDMA schemes to increase the rate.

\section{APPENDIX}

Proof: We prove that the two sums in expression (5) of the SINR for the Matched Filter converge in expectation (almost sure convergence can also be shown but is not detailed for sake of space limitation) to obtain Proposition 1. Namely,

$$
\begin{gathered}
\sum_{i=1}^{N}\left|h_{i 1}\right|^{2}\left|w_{i 1}\right|^{2} \underset{N \rightarrow \infty}{\sim} \xi_{1} \\
\sum_{k=2}^{K}\left|\sum_{i=1}^{N} h_{i 1}^{*} h_{i k} w_{i 1}^{*} w_{i k}\right|^{2} \underset{N \rightarrow \infty}{\sim} \alpha \xi_{1}\left(\varrho-\eta_{1}\right)
\end{gathered}
$$

Note that (7) is immediate:

$$
\mathbb{E}_{w}\left[\sum_{i=1}^{N}\left|h_{i 1}\right|^{2}\left|w_{i 1}\right|^{2}\right]=\frac{1}{N} \sum_{i=1}^{N}\left|h_{i 1}\right|^{2} \underset{N \rightarrow \infty}{\longrightarrow} \xi_{1}
$$

The expression $\mathbb{E}_{w}\left[\left|\sum_{i=1}^{N} h_{i 1}^{*} h_{i k} w_{i 1}^{*} w_{i k}\right|^{2}\right]$ is equal to:

$$
\begin{aligned}
& \sum_{i=1}^{N} \sum_{l=1}^{N} h_{i 1}^{*} h_{i k} h_{l 1} h_{l k}^{*} \mathbb{E}_{w}\left[w_{i 1}^{*} w_{i k} w_{l 1} w_{l k}^{*}\right] \\
& =\frac{1}{N(N+1)} \sum_{i=1}^{N}\left|h_{i 1}\right|^{2}\left|h_{i k}\right|^{2} \\
& \quad-\frac{1}{N\left(N^{2}-1\right)} \sum_{i=1}^{N} \sum_{l \neq i} h_{i 1}^{*} h_{l 1} h_{i k} h_{l k}^{*}
\end{aligned}
$$

using the fact that

$$
\begin{aligned}
\mathbb{E}\left[\left|w_{i 1}\right|^{2}\left|w_{i k}\right|^{2}\right] & =\frac{1}{N(N+1)}, k>1 \\
\mathbb{E}\left[w_{i 1}^{*} w_{i k} w_{l 1} w_{l k}^{*}\right] & =-\frac{1}{N\left(N^{2}-1\right)}, k>1, i \neq l
\end{aligned}
$$

When $N$ becomes large, (9) and (10) tend respectively to

$$
\frac{1}{N+1} \frac{1}{W} \int_{-\frac{W}{2}}^{\frac{W}{2}}\left|h_{1}(f)\right|^{2}\left|h_{k}(f)\right|^{2} d f
$$

and

$$
\frac{1}{N+1} \frac{1}{W} \int_{-\frac{W}{2}}^{\frac{W}{2}} h_{1}^{*}(u) h_{k}(u) d u \frac{1}{W} \int_{-\frac{W}{2}}^{\frac{W}{2}} h_{1}(v) h_{k}^{*}(v) d v
$$

It is then rather straightforward to obtain (8).

\section{REFERENCES}

[1] S. Shamai and S. Verdu, "The Impact of Frequency-Flat Fading on the Spectral Efficiency of CDMA," IEEE Trans. on Information Theory, pp. 1302-1326, May 2001.

[2] D.N.C Tse and S. Hanly, "Linear Multi-user Receiver: Effective Interference, Effective Bandwidth and User Capacity," IEEE Trans. on Information Theory, pp. 641-657, Mar. 1999.

[3] M. Debbah, W. Hachem, P. Loubaton, and M. de Courville, "MMSE Analysis of Certain Large Isometric Random Precoded Systems," IEEE Transactions on Information Theory, Volume: 49 Issue: 5, Page(s): 1293 -1311, May 2003.

[4] J.M. Chaufray, W. Hachem, and Ph. Loubaton, "Asymptotical Analysis of Optimum and Sub-Optimum CDMA Downlink MMSE Receivers," in IEEE International Symposium on Information Theory, Lausanne, Switzerland, July 2002.

[5] M. Debbah, P. Loubaton, and M. de Courville, "Asymptotic Performance of Successive Interference Cancellation in the Context of Linear Precoded OFDM," IEEE Transactions on Communications, vol. 52, No 9 September 2004.

[6] F. Hiai and D. Petz, The Semicircle Law, Free Random Variables and Entropy, vol. 77 of Mathematical Surveys and Monographs, AMS, 2000.

[7] D. Petz and J. Réffy, "On asymptotics of large Haar distributed unitary matrices," Periodica Math. Hungar, vol. 49, pp. 103-117, 2004.

[8] S. Verdu and S. Shamai, "Spectral Efficiency of CDMA with Random Spreading ," IEEE Trans. on Information Theory, pp. 622-640, Mar. 1999.

[9] K. Fazel and L. Papke, "On the Performance of Convolutionally-Coded CDMA/OFDM for Mobile Communication System," in proceedings of IEEE PIMRC, Yokohama, Japan, Sept. 1993, pp. 468-472.

[10] Jürgen Lindner, "MC-CDMA and its Relation to General Multiuser/Multisubchannel Transmission Systems," in International Symposium on Spread Spectrum Techniques \& Applications, Mainz, Germany, Sept. 1996, pp. 115-121.

[11] J.A.C. Bingham, "Multicarrier Modulation for Data Transmission: An Idea Whose Time Has Come," IEEE Communications Magazine, vol. 28, no. 5, pp. 5-14, May 1990.

[12] R. Gray, Toeplitz and Circulant Matrices, Stanford University, Palo Alto, 1st edition, 1977.

[13] W. Hachem, "Simple Polynomial MMSE Receivers for CDMA Transmissions on Frequency Selective Channels," IEEE Trans. on Information Theory, pp. 164 - 172, January 2004.

[14] S. Verdú, Multiuser Detection, Cambridge University Press, 1998.

[15] R. Müller and S. Verdu, "Design and Analysis of Low-Complexity Interference Mitigation on Vector Channels," IEEE Journal on Selected Areas in Communications, pp. 1429-1441, Aug. 2001.

[16] N. Bonneau, M. Debbah, and E. Altman, "When to Synchronize in Uplink CDMA," in print.

[17] V. L. Girko, "Theory of Random Determinants," Kluwer Academic Publishers, Dordrecht, The Netherlands, 1990.

[18] A. Tulino, L. Li, and S. Verdu, "Spectral Efficiency of Multicarrier CDMA," IEEE Trans. on Information Theory, pp. 479 - 505, February 2005. 